\section{QUALIFICAÇÃO DA DOR EM TRABALHADORAS DE UMA MALHARIA NO SUL DO BRASIL}

\author{
Qualification of pain in women workers of a textile factory in \\ the south of Brazil
}

\author{
Cualificación del dolor de trabajadoras de una tienda de mallas \\ del sur de Brasil
}

\section{RESUMO}

Objetivo: Analisar e qualificar a dor em mulheres de uma malharia do sul do Brasil. Métodos: Estudo transversal, descritivo, com abordagem quantitativa, em que foram entrevistadas 15 trabalhadoras em um único dia de maio de 2012. As avaliações para qualificação e análise da dor aconteceram por meio de três questionários: multidimensional adaptado, inventário para dor de Wisconsin (análise da dor) e o questionário de McGill (qualificação). Para análise dos dados, foi realizada a estatística descritiva, com o calculo da média, desvio padrão, percentual e números absolutos. Resultados: As participantes apresentavam média de idade de 33,4 $( \pm 14,9)$ anos e trabalhavam 9 horas diárias. Referente à postura dolorosa no posto de trabalho, observou-se maior queixa em ombros abduzidos e cotovelos flexionados $(n=7,46,6 \%)$. Os locais anatômicos dolorosos mais frequentes foram ombros $(n=8,22,2 \%)$, coluna cervical $(n=7,19,4 \%)$ e cotovelos $(n=7,19,4 \%)$. A análise da dor pelo inventário de Wisconsin, classificada por meio da Escala Visual Analógica de Dor (EVA), demonstrou interferência moderada no trabalho, sono, humor, apreciação da vida e na atividade geral. De acordo com o questionário de $M c G i l l$, houve destaque para o nível sensorial, com maior frequência para dor latejante $(n=10,50 \%)$, agulhada $(n=4,20 \%)$ e formigamento $(n=4,20 \%)$. Conclusão: A amostra apresentou alta frequência de dor nos ombros, cotovelos, coluna cervical e sintomatologia dolorosa considerada moderada na maioria dos itens avaliados, o que pode levar a uma interferência importante nas atividades laborais e do cotidiano.

Descritores: Saúde do Trabalhador; Indústria Têxtil; Fisioterapia; Mulheres; Dor.

\section{ABSTRACT}

Objective: To analyze and qualify pain in women workers of a textile factory in the south of Brazil. Methods: Cross-sectional descriptive study, with quantitative approach, in which 15 women workers were interviewed on a single day in May 2012. The assessments for pain qualification and analysis were performed by means of three instruments: multidimensional adapted questionnaire, Wisconsin Pain inventory (pain analysis) and McGill Pain questionnaire (qualification). For data analysis, descriptive statistics was employed, by calculating the average, standard deviation, percentage and absolute numbers. Results: The participants' mean age was 33.4 ( \pm 14.9) and they worked 9 hours a day. As for the painful posture in the workplace, the major complaint regarded abducted shoulders and flexed elbows $(n=7,46.6 \%)$. The most commonly painful anatomic sites were shoulders $(n=8,22.2 \%)$, cervical spine $(n=7,19.4 \%)$ and elbows $(n=7,19.4 \%)$. Pain analysis through the Wisconsin inventory, classified by the Visual Analogue Pain Scale (VAS), demonstrated moderate interference with work, sleep, mood, enjoyment of life and general activity. According to McGill questionnaire, the sensory level stood out, with highest frequencies to throbbing pain $(n=10,50 \%)$, needled $(n=4,20 \%)$ and tingling pain $(n=4,20 \%)$. Conclusion: The sample showed high frequency of pain in shoulders, elbows and cervical spine, in addition to painful symptomatology regarded moderate in most of the evaluated items, which might lead to significant interference with the labor activities and daily life.

Descriptors: Occupational Health; Textile Industry; Physical Therapy Specialty; Women; Pain.
Artigo Original
1) Universidade Comunitária da Região de Chapecó - UNOCHAPECÓ - Chapecó (SC) - Brasil
Recebido em: 01/10/2015 Revisado em: 22/12/2015 Aceito em: 12/02/2016 


\section{RESUMEN}

Objetivo: Analizar y cualificar el dolor de mujeres de una tienda de mallas del sur de Brasil. Métodos: Estudio transversal y descriptivo de abordaje cuantitativo en el cual se entrevistaron 15 trabajadoras en un único día del mes de mayo de 2012. Las evaluaciones para la cualificación y el análisis del dolor se dieron a través de tres cuestionarios: el multidimensional adaptado, el inventario para el dolor de Wisconsin (análisis del dolor) y el cuestionario de McGill (cualificación). Para el análisis de los datos se realizó la estadística descriptiva con el cálculo de la media, la desviación típica, el porcentual y los valores absolutos. Resultados: Las participantes presentaron la media de edad de $33,4( \pm 14,9)$ años y 9 horas al día de trabajo. Respecto la postura de dolor en el sector de trabajo, se observó más quejas en los hombros en abducción y los codos en flexión ( $n=7,46,6 \%)$. Los puntos anatómicos de dolor más frecuentes fueron los hombros $(n=8,22,2 \%)$, la columna cervical $(n=7,19,4 \%)$ y los codos (n=7, 19,4\%). El análisis del dolor a través del inventario de Wisconsin clasificado por la Escala Visual Analógica de Dolor (EVA) ha demostrado interferencia moderada con el trabajo, el sueño, el humor, la apreciación de la vida y la actividad general. Según el cuestionario de McGill hubo importancia para el nivel sensorial con más frecuencia para el dolor latigante ( $n=10,50 \%)$, en agujada $(n=4,20 \%)$ y hormigueo $(n=4,20 \%)$. Conclusión: La muestra presentó elevada frecuencia para el dolor en los hombros, los codos, la columna cervical y sintomatología de dolor moderada para la mayoría de items evaluados lo que puede llevar a una interferencia importante en las actividades laborales y del cotidiano.

Descriptores: Salud Laboral; Industria Textil; Fisioterapia; Mujeres; Dolor.

\section{INTRODUÇÃO}

Durante muito tempo, tem-se realizado grandes esforços para diminuir as desigualdades sociais entre os gêneros. Com isso, as mulheres, cada vez mais, participam ativamente do mercado de trabalho, o que, por sua vez, tem proporcionado uma dupla e/ou tripla jornada de trabalho. Além disso, ainda existe uma disparidade nos salários entre os sexos, acarretando em maiores sofrimentos emocionais e problemas ligados à saúde, como variações hormonais, sedentarismo, fadiga muscular e depressão $0^{(1,2)}$.

Os trabalhadores estão expostos a diversas modalidades e intensidades de riscos no ambiente de trabalho, os quais aumentam à medida que cresce a utilização de recursos tecnológicos associados às diferentes formas de controle e organização, levando à insatisfação e elevação nos perfis de morbidade da classe trabalhadora. Sabe-se que esses riscos produzem vários sintomas, sendo o principal a dor ${ }^{(3)}$.

Constituindo a categoria mais populosa na indústria têxtil, as mulheres são as mais acometidas pelas doenças laborais, sendo estas, na sua maioria, problemas musculoesqueléticos, alterações respiratórias e quadro de estresse $^{(4)}$. Os distúrbios osteomusculares relacionados ao trabalho (DORT) resultam em dor persistente, perda da capacidade funcional, incapacidade laboral e apresentam causa multifatorial, incluindo fatores físicos, organizacionais e individuais ${ }^{(5,6)}$.

Diferentes métodos têm sido utilizados para avaliar a percepção da dor, sendo esta uma experiência multidimensional, com componentes sensoriais, afetivos e cognitivo-avaliativos que se correlacionam, colaborando para a resposta dolorosa ${ }^{(5)}$. Medidas unidimensionais de intensidade, em que a Escala Visual Analógica (EVA) é a mais utilizada, e escalas multidimensionais, como o questionário de McGill e o inventário de Wisconsin, são exemplos de métodos que conseguem quantificar e qualificar os diversos componentes vinculados à dor ${ }^{(7,8)}$.

Estudos afirmam que o uso de escalas pode ser mais efetivo para avaliar a dor, pois se sabe que existe relação entre ela e as qualidades sensoriais, afetivas e temporais. Desse modo, o uso de escalas possibilita estimar as repercussões provocadas por essa sintomatologia no cotidiano e nas atividades laborais dos trabalhadores ${ }^{(9-11)}$.

Além disso, vale destacar que, segundo os dados do Instituto Nacional do Seguro Social (INSS), foram registrados, em 2013, 717.900 acidentes e doenças do trabalho, com consequente afastamento de 610.804 trabalhadores por incapacidade temporária. Foi observado um acréscimo de 0,55\% em relação ao ano de 2012, e entre esses registros contabilizaram-se 15.226 doenças relacionadas ao trabalho ${ }^{(12)}$.

As doenças ocupacionais proporcionam grandes consequências sociais e econômicas, tanto para o Estado como para as empresas e o próprio indivíduo. Para o Estado, um alto gasto com pagamentos de benefícios previdenciários, tratamento e reabilitação. Para a empresa, perda na produção e na qualidade no serviço pela diminuição do número de funcionários e horas de trabalho; e para o indivíduo, sofrimento, incapacidade para o trabalho, para a atividade do cotidiano e vida social ${ }^{(13)}$.

Diante desse contexto, as doenças ocupacionais podem ser consideradas como um problema de saúde pública, sendo necessárias ações políticas preventivas e protetivas contra os riscos ocupacionais e aspectos relacionados à saúde do trabalhador para reduzir essa alta prevalência. Assim, este estudo teve como objetivo analisar e qualificar a dor em mulheres de uma malharia do sul do Brasil.

\section{MÉTODOS}

Este estudo é considerado de cunho quantitativo, descritivo e transversal. A população de mulheres 
trabalhadoras de uma malharia do oeste catarinense era de 19, mas a amostra do presente estudo foi composta por 15 mulheres com média de idade de 33,4 $( \pm 14,9)$ anos. A coleta de dados foi realizada em um único dia de maio de 2012 e ocorreu no próprio local de trabalho, onde as participantes responderam, de forma autoaplicável, três questionários.

A produção têxtil da malharia investigada é dividida em três setores - corte, costura e passar as peças: uma pessoa realiza o corte e outra passa as peças, ambas permanecendo na posição ortostática estática; as demais realizam a costura, mantendo-se na posição sentada durante toda a jornada. Observa-se que, em todos os setores, as atividades realizadas compõem-se de movimentos repetitivos, exigindo principalmente dos membros superiores, além de utilizarem mobiliário ergonomicamente inadequado. A jornada de trabalho tem duração de 9 horas diárias.

Como critérios de inclusão, foram considerados: estar presente no momento da coleta de dados, ser alfabetizada e responder aos três instrumentos de avaliação de forma completa. Dentro desse contexto, foram excluídas quatro mulheres da amostra, devido à sua ausência no dia estabelecido ou recusa da participação para a coleta de dados.

A avaliação da dor foi realizada mediante a aplicação de três questionários: McGill, inventário para dor de Wisconsin e questionário multidimensional, sendo realizada em uma única visita à empresa, previamente agendada. Após a chegada e explicação do estudo, foi entregue às entrevistadas os três questionários, os quais foram auto aplicados. A duração do preenchimento completo de todos os instrumentos foi de aproximadamente 20 minutos e em seguida a participante retornava às suas atividades laborais.

O questionário de McGill consiste em um instrumento multidimensional, com 78 descritores divididos em quatro grupos, sendo eles: sensorial (subgrupo de 1 a 10), afetivo (subgrupo de 11 a 15), avaliativo (subgrupo 16) e miscelânea (subgrupo 17 a 20). Para a aplicação desse questionário, pede-se que o indivíduo escolha uma palavra de cada

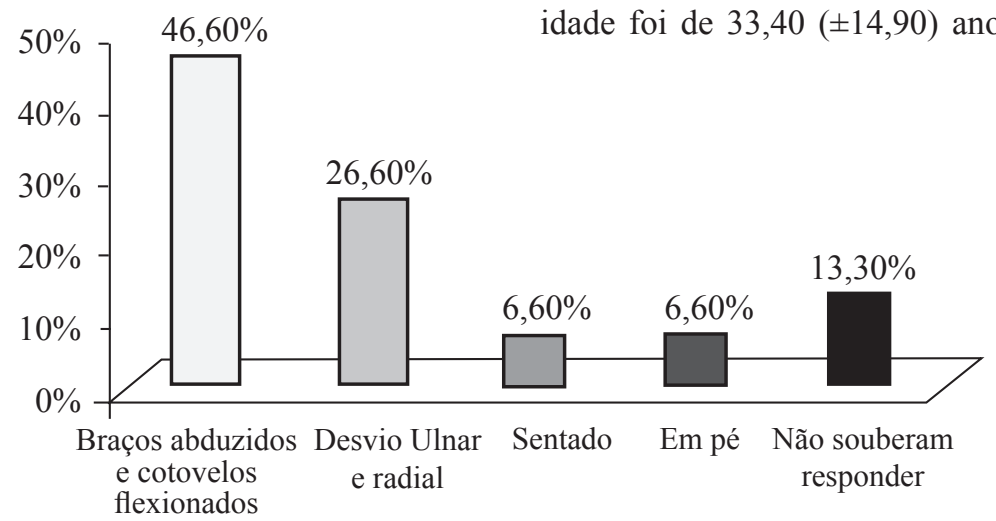

Figura 1 - Caracterização da postura dolorosa no trabalho. Chapecó, SC, 2012. subcategoria, sendo permitida a opção de não escolher nenhuma. A análise é feita por categorias, através da soma dos valores associados às palavras escolhidas (número de descritores), e o índice de dor é obtido através da somatória dos valores de intensidade dos descritores escolhidos ${ }^{(14)}$.

Sobre a análise da intensidade geral da dor na atividade laboral e extralaboral, foi usado o inventário para dor de Wisconsin $^{(15)}$, composto por um diagrama corporal que faz a caracterização e localização da queixa, questões relacionadas à intensidade, descrição do alívio alcançado e da impressão do paciente a respeito da sua queixa álgica. No entanto, as respostas foram assinaladas na EVA, que apresenta como classificação: de 0 a 2 para dor leve, 3 a 7 para dor moderada e 8 a 10 para dor intensa.

Com intuito de obter dados relevantes sobre as condições de trabalho, foi entregue um questionário multidimensional, adaptado para o presente estudo, que continha questões sobre cargo que ocupa, posição adotada durante a tarefa que desempenha, realização de atividades extralaborais e prática de atividade física ${ }^{(16)}$. Além disso, vale ressaltar que, para facilitar a localização dolorosa, foi apresentada uma figura ilustrativa do corpo humano ${ }^{(17)}$.

Para apresentação dos dados, foi utilizada estatística descritiva, com os dados sendo representados com valores de média, desvio padrão, percentuais e números absolutos, com o auxílio do programa SPSS, versão 13.0.

O estudo foi iniciado após aprovação pelo Comitê de Ética em Pesquisa envolvendo Seres Humanos, da Universidade Comunitária da Região de Chapecó, com o protocolo $n^{\circ} 355 / 12$, atendendo aos requisitos da Resolução 466/12 e suas complementares do Conselho Nacional de Saúde, e autorização da empresa onde foi realizado o estudo. A participação da população ocorreu mediante leitura, compreensão e autorização por escrito do Termo de Consentimento Livre e Esclarecido.

\section{RESULTADOS}

Com relação à caracterização da amostra, a média de idade foi de 33,40 $( \pm 14,90)$ anos, a maioria era casada e 
tinha filhos ( $\mathrm{n}=10,66,7 \%)$, com segundo grau completo de escolaridade ( $\mathrm{n}=7,46,6 \%)$. Como patologia associada, $40 \%$ $(n=6)$ tinham hipertensão arterial e 6,6\% $(n=1)$, enxaqueca e hipertrigliceredemia. Quanto à prática de atividade física, $86,6 \%(\mathrm{n}=13)$ foram classificadas como sedentárias, pois não realizavam nenhum tipo de exercício físico. Sobre a função laboral, $86,6 \%(\mathrm{n}=13)$ das mulheres trabalhavam no setor de costura, $80 \%(\mathrm{n}=12)$ realizavam horas-extras e $80 \%(n=12)$ faziam pausa ao menos uma vez por turno. Foi observado que, além das atividades laborais, todas as mulheres desempenham também atividades domiciliares.
A Figura 1 apresenta os dados referentes à postura dolorosa no posto de trabalho. Observa-se que a maioria relatou a postura mantida de ombros abduzidos, com cotovelos flexionados, usada na atividade de costura.

A Figura 2 apresenta as frequências dos locais anatômicos dolorosos, a partir do inventário de Wisconsin. Como pode ser observado, ombro, cotovelo e coluna cervical foram os locais mais acometidos pelo quadro álgico.

A Tabela I mostra as variáveis e médias de dor referidas pelas trabalhadoras na escala de 0 (sem queixa) a 10 (o pior quadro). Observa-se para todas as variáveis estudadas

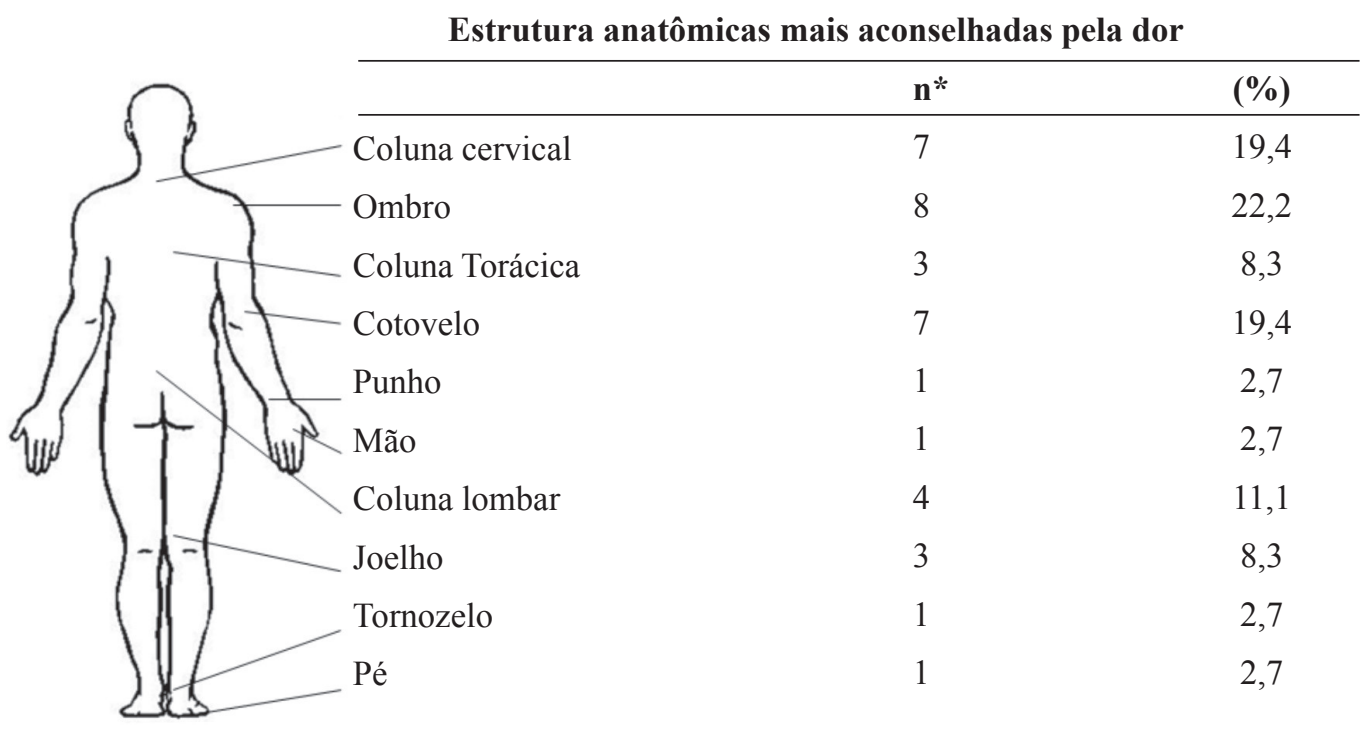

Figura 2 - Frequência dos locais anatômicos dolorosos acometidos. Chapecó, SC, 2012.

*= um mesmo indivíduo pode relatar dor em mais de um local anatômico.

Fonte imagem: MAGEE DJ ${ }^{(15)}$.

Tabela I - Variáveis de dor referida pelas trabalhadoras segundo inventário de dor de Wisconsin. Chapecó, SC, 2012.

\begin{tabular}{lccc}
\hline Variáveis de dor & Média \pm DP* & Mínimo-Máximo & Classificação \\
\hline Pior dor nas últimas 24 horas & $3,6 \pm 3,6$ & $0,0-10,0$ & Moderada \\
Dor mais fraca sentida nas últimas 24 horas & $2,5 \pm 1,6$ & $0,0-5,0$ & Moderada \\
Média da dor sentida & $3,5 \pm 2,1$ & $0,0-5,0$ & Moderada \\
Dor no momento da entrevista & $2,1 \pm 3,2$ & $0,0-10,0$ & Moderada \\
Interferência da dor na atividade geral nas últimas 24 horas & $3,9 \pm 2,8$ & $0,0-8,0$ & Moderada \\
Interferência da dor no relacionamento com as pessoas & $0,8 \pm 1,4$ & $0,0-4,0$ & Leve \\
Interferência da dor no trabalho & $3,6 \pm 3,1$ & $0,0-8,0$ & Moderada \\
Interferência da dor na habilidade de caminhar & $1,8 \pm 2,6$ & $0,0-7,0$ & Leve \\
Interferência da dor no sono & $3,8 \pm 3,9$ & $0,0-10,0$ & Moderada \\
Interferência da dor no humor & $3,0 \pm 2,9$ & $0,0-8,0$ & Moderada \\
Interferência da dor na apreciação da vida & $2,9 \pm 3,3$ & $0,0-9,0$ & Moderada \\
\hline
\end{tabular}

DP: desvio padrão. 
Tabela II - Caracterização da dor em trabalhadoras de uma malharia de acordo com o questionário de McGill. Chapecó, SC, 2012.

\begin{tabular}{llcc}
\hline Categoria & Descritor & $\mathbf{n}$ & \% \\
\hline & Torção & 1 & 5,0 \\
& Mal localizada & 2 & 10,0 \\
Latejante & 10 & 50,0 \\
Sensorial & Formigamento & 4 & 20,0 \\
& Agulhada & 4 & 20,0 \\
& Ferroada & 3 & 15,0 \\
& Cólica & 1 & 5,0 \\
& Sensível & 1 & 5,0 \\
& Queimação & 3 & 15,0 \\
Afetiva & Dolorida & 1 & 5,0 \\
\multirow{2}{*}{ Avaliativo } & Pontada & 2 & 10,0 \\
& Fisgada & 1 & 5,0 \\
& Cansativa & 6 & 30,0 \\
Miscelânea & Enjoada & 2 & 10,0 \\
& Atormenta & 1 & 5,0 \\
& Desgastante & 1 & 5,0 \\
& Que incomoda & 5 & 25,0 \\
& Adormece & 3 & 15,0 \\
& Irradia & 1 & 5,0 \\
& Espalha & 1 & 5,0 \\
& Dá náuseas & 4 & 20,0 \\
\hline
\end{tabular}

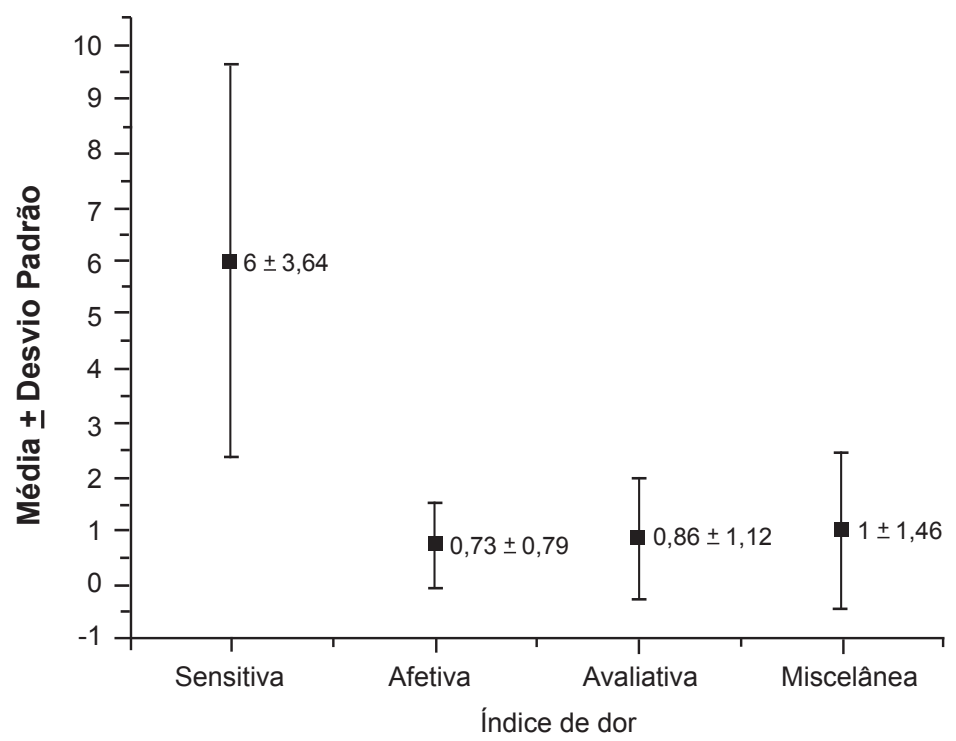

Figura 3 - Índice de dor referido pelas trabalhadoras por meio do questionário de McGill. Chapecó, SC, 2012.

o nível de dor variou entre leve a moderado e que afeta principalmente as atividades gerais e o sono.

A Tabela II refere-se aos descritores selecionados no questionário de dor de McGill. Foram escolhidos descritores em todas as categorias, tendo destaque para o nível sensorial.
Os descritores que obtiveram maior frequência foram: latejante (50\%), agulhada (20\%) e formigamento (20\%).

A Figura 3 mostra o índice de dor de acordo com o questionário de McGill. Observou-se que a média da dor sensorial foi a mais alta quando comparada com a média da dor afetiva, avaliativo ou miscelânea. 


\section{DISCUSSÃO}

O presente estudo buscou qualificar a dor em mulheres de uma malharia no município do Sul do país. Os resultados demonstraram alta frequência de dor nos ombros, cotovelo e coluna cervical, apresentando principalmente causa mecânica, térmica e espacial, além disso, a dor foi relacionada às posturas mantidas, assim como com o sedentarismo e a dupla jornada de trabalho.

Um estudo sobre trabalhadoras na indústria têxtil mostra que o estilo de vida aliado a condições inadequadas de trabalho podem favorecer o aparecimento de doenças, assim, as políticas de saúde do trabalhador devem vislumbrar ações de promoção da saúde e melhores condições de trabalho ${ }^{(18)}$.

As trabalhadoras analisadas na presente pesquisa relataram que, além da jornada de trabalho na malharia, desempenham atividades no âmbito domiciliar, corroborando com outra pesquisa que relata o aumento da participação feminina no mercado de trabalho, fato que tem exigido das mulheres múltiplos papéis ${ }^{(19)}$. Além disso, há indícios na literatura de que o tempo despendido pelas mulheres em afazeres domésticos é sempre maior que o dos seus cônjuges ${ }^{(20)}$.

O mercado de trabalho brasileiro vem sendo marcado pela constante atuação do gênero feminino desde a última década do século XX, e o perfil dessas trabalhadoras é de mulheres mais velhas, casadas e mães, porém, não deixam seus afazeres domésticos e o cuidado com os filhos e outros familiares, voltando-se tanto para o trabalho quanto para a família, fazendo permanecer o modelo de família tradicional. A grande consequência disso é a sobrecarrega dessas trabalhadoras ${ }^{(21)}$, levando-as a adquirir doenças relacionadas ao trabalho, devido à jornada exercida ${ }^{(22)}$.

Um estudo realizado numa indústria têxtil de Divinópolis, utilizando observações dos ambientes de trabalho e entrevistas semiestruturadas, concluiu que a maior inclusão de mulheres no mercado de trabalho tem aumentado as tarefas femininas e tornado as jornadas de trabalho extensas, cansativas e até mesmo estressantes, designando a sobrecarga de trabalho e favorecendo patologias e disfunções musculoesqueléticas relacionadas a esforços repetitivos, tendo como consequência interferências na produtividade, no psicológico e no relacionamento familiar(23).

Dessa forma, o estresse físico proporcionado pela dupla jornada de trabalho e vivenciado intensamente pelas mulheres tem acarretado em inúmeras patologias, além de exercer importante impacto na qualidade de vida ${ }^{(24)}$. Além disso, fatores como biótipo, composição corporal e hormonal também podem ser envolvidos no aparecimento do sintoma doloroso nas mulheres ${ }^{(24,25)}$.
A indústria têxtil apresenta maior presença do sexo feminino $^{(16)}$, por ser considerada mais precisa e delicada e pelo fato de que, no imaginário masculino, costura é coisa para mulher ${ }^{(25)}$, condição essa que corrobora com os dados do presente estudo, no qual observou-se que a frente de trabalho da malharia era composta apenas por mulheres. Além disso, vale destacar que as indústrias têxteis foram pioneiras em abrigar a mão de obra feminina durante a distribuição sexual do trabalho na Revolução Industrial ${ }^{(26)}$.

Das entrevistadas no atual estudo, $80 \%$ afirmaram realizar horas extras. Esse resultado concorda com outro estudo $^{(27)}$, o qual relata que, com os avanços tecnológicos, o processo e a organização do trabalho mudaram, pois foram incorporadas novas formas de gestão, visando a produtividade e a qualidade do produto, o que acarretou em aumento do ritmo de trabalho e, consequentemente, em horas extras, levando ao afastamento da vida social. Além disso, foi observado que, em $79,6 \%$ dos profissionais da indústria têxtil, a realização de horas extras era rotineira ${ }^{(16)}$.

De acordo com os resultados, pôde-se constatar que a maior parte da amostra não praticava atividade física. Em um estudo envolvendo pacientes com lombalgia crônica, $64,7 \%$ não realizavam nenhuma prática esportiva ${ }^{(28)}$; e em outro estudo que analisava aspectos relacionados ao trabalho e às condições de saúde sobre a sintomatologia dolorosa em profissionais da indústria têxtil, verificou-se maior prevalência de dor em trabalhadores que relataram ter alguma patologia e não realizavam atividade física ${ }^{(16)}$. Desse modo, pode-se sugerir que a prática regular de exercícios físicos está associada ao bem-estar físico, mental e social, proporcionando redução da intensidade da dor ${ }^{(29-31)}$.

Vale destacar que um programa de ginástica laboral diária na empresa, composto por exercícios de aquecimentos no início do turno e duas pausas para alongamentos, fazse de extrema importância, posto que há associação entre ginástica laboral e alívio da fadiga física e mental ${ }^{(22)}$.

A postura sentada por longos períodos exige um trabalho muscular estático, pois ela apresenta maior prevalência de sintomatologia dolorosa se comparada com a dinâmica, podendo desenvolver processos inflamatórios e dor ${ }^{(16,32)}$. Na presente pesquisa, o setor de costura era composto por uma maioria de mulheres $(86,6 \%)$, que permanecia na postura sentada estática e realizava movimentos repetitivos de membros superiores, sendo este o local anatômico com maior presença de queixas. Em um estudo com 176 profissionais que exerciam atividades de trabalho sedentárias, os autores revelaram que existe uma relação direta dos movimentos de repetição com a origem de muitos problemas musculoesqueléticos, em decorrência das contrações contínuas e aumento da pressão intramuscular, além da interrupção do aporte sanguíneo e compressão de feixes nervosos, que levam ao sofrimento muscular crônico ${ }^{(33)}$. 
Deve-se também pensar que a operação de máquinas de costura demanda o uso repetitivo e coordenado do tronco, membros superiores e inferiores das trabalhadoras, que permanecem em postura sentada por um longo período de tempo, proporcionando elevada sobrecarga física e expondo-as a sintomas dolorosos, como fadiga muscular e até mesmo lesões, principalmente na região lombar e nos membros inferiores ${ }^{(26)}$.

As condições biomecânicas e ergonômicas inadequadas devem ser levadas em consideração ao se analisarem os fatores de risco ocupacionais que ocasionam a elevada ocorrência de queixas álgicas. No entanto, as dores nas regiões intermediária e inferior da coluna vertebral e nos membros inferiores foram as mais citadas em estudos realizados com costureiras, discordando dos dados obtidos no presente estudo ${ }^{(16,34)}$.

Vale destacar que os resultados apontaram ombros $(22,20 \%)$, cotovelos $(19,40 \%)$ e coluna cervical $(19,40 \%)$, respectivamente, como sendo os locais anatômicos que apresentaram maior frequência dolorosa. Tal fato pode ser justificado pela postura mantida pelas entrevistadas e pelo gesto biomecânico adotado, sendo caracterizados por cabeça anteriorizada, ombros abduzidos e cotovelos flexionados. Em comparação, um estudo com funcionários de uma indústria têxtil observou alta prevalência de dor na coluna vertebral (cervical e torácica), pernas e ombros, respectivamente ${ }^{(16)}$. Além disso, outra pesquisa abordando trabalhadores de indústria têxtil destacou como queixas principais a lombalgia $(28,8 \%)$ e a cervicalgia $(22,0 \%)$, devido ao esforço físico associado com a repetição dos movimentos ${ }^{(35)}$.

Por meio do inventário para dor proposto por Wisconsin, foi possível avaliar a intensidade geral da dor e como ela interfere nas atividades cotidianas e laborais, apresentando maior importância nas variáveis atividades gerais, trabalho, sono, humor e apreciação da vida, com intensidade leve a moderada. Ao utilizar o mesmo instrumento para avaliação da dor de membros inferiores de costureiros, observou-se alta incidência sintomatológica moderada, apresentando intensidade $(6 \pm 2,3)^{(34)}$.

De acordo com o questionário de $M c$ Gill, os descritores com maior ocorrência foram da categoria sensitiva que se refere às propriedades mecânicas, térmicas, de vividez e espaciais da dor, como "latejante", "formigamento" e "agulhada". A categoria afetiva diz respeito aos aspectos de tensão, medo e respostas neurovegetativas, mas a palavra "cansativa" foi mais utilizada. Na categoria avaliativa, que permite o indivíduo expressar a avaliação global da experiência dolorosa, a expressão mais citada foi "que incomoda". Já a categoria miscelânea, de caráter mais sensitivo, o descritor mais mencionado foi "dá náuseas". Outro estudo com a mesma população mostrou que $85 \%$ das costureiras relataram dor na região do pescoço. Esse desconforto foi classificado como "agulhada" em 47\% dos casos e como "formigamento" em $29 \%$; além disso, a região do ombro foi referida por $50 \%$ das costureiras, sendo classificada como "formigamento" (50\%) e "sensação de peso" $(30 \%)^{(36)}$.

Corroborando com os achados do presente estudo, um estudo registrou as características das queixas dolorosas em pacientes que buscaram a clínica de fisioterapia, obtidas pelo questionário da dor de $\mathrm{McGill}$, encontrando a dor "cansativa" (57,4\%), "adormecida" $(42,6 \%)$ e a dor "que incomoda" $(30,9 \%)^{(37)}$. Por outro lado, discordando dos resultados, em um estudo sobre dor e funcionalidade em indivíduos com lombalgia, a categoria sensitiva obteve maior frequência para a dor "que queima" $(70,6 \%)$ e "em pontada" (64,7\%), no entanto, no presente estudo, a dor "latejante" foi a mais frequente, e na categoria afetiva, observou-se um maior predomínio de dor "enjoada" $(76,5 \%)$, sendo "cansativo" o descritor mais relatado pelas mulheres do presente estudo ${ }^{(30)}$.

Por fim, vale destacar algumas limitações do estudo. A amostra foi composta por um número pequeno de trabalhadoras, fato que dificultou a análise estatística avançada. Além disso, não foram encontrados na literatura pertinente estudos referentes a esse tema que apresentassem o uso de questionários multidimensionais da dor, o que poderia ter enriquecido a discussão do estudo. Apesar disso, com os resultados obtidos, espera-se contribuir para melhor entendimento da sintomatologia dolorosa de mulheres trabalhadoras de uma malharia, além de auxiliar no planejamento e adequação de programas preventivos ou de atividade física que possam colaborar para a manutenção da saúde e redução das queixas álgicas dessa população.

Para isso, é imprescindível que as indústrias reconheçam a necessidade de um trabalho educativo de promoção da saúde para os trabalhadores, e que estes também assumam suas responsabilidades e compreendam o seu papel na prevenção de agravos, tanto os relacionados ao trabalho como aos hábitos de vida.

\section{CONCLUSÃO}

Verificou-se alta frequência de dor nos ombros, cotovelos e coluna cervical, apresentando principalmente origem sensorial, podendo estar relacionada às posturas mantidas, bem como à inatividade física e à dupla jornada de trabalho nas trabalhadoras investigadas. Constatou-se, dessa forma, que a sintomatologia dolorosa considerada moderada na maioria dos itens avaliados interfere de maneira significativa nas atividades laborais e do cotidiano, prejudicando o desempenho dessas profissionais. 


\section{REFERÊNCIAS}

1. Gomes KRO, Tanakka AC. Morbidade referida e uso dos serviços de saúde por mulheres trabalhadoras, município de São Paulo. Rev Saúde Pública. 2003;37(1):75-82.

2. Leone ET, Baltar P. A mulher na recuperação recente do mercado de trabalho brasileiro. Rev Bras Estud Popul. 2008;25(2):233-49.

3. Leite PC, Silva A, Mereghi MAB. A mulher trabalhadora de enfermagem e os distúrbios osteomusculares relacionados ao trabalho. Rev Esc Enferm USP. 2007;41(2):287-91.

4. Pernambucano AP. Saúde e Trabalho: o caso dos trabalhadores da indústria do vestuário de Formiga MG [dissertação]. Divinópolis: Universidade Estadual de Minas Gerais/Curso de Mestrado em Educação, Cultura e Organizações Sociais; 2007.

5. Walsh IAP, Oishi J, Coury HJCG. Aspectos clínicos e funcionais de distúrbios musculoesqueléticos entre trabalhadores ativos. Rev Saúde Pública. 2008;42(1):108-16.

6. Caetano VC, Cruz DT, Leite ICG. Perfis dos pacientes e características do tratamento fisioterapêutico aplicado aos trabalhadores com LER/DORT em Juiz de Fora, MG. Fisioter Mov. 2010;23(3):451-60.

7. Rocha CED, Martins MI, Foss MH, Santos R Júnior, Dias LC, Forni JE et al. Melhorar da qualidade de vidade de pacientes com dor neuropática utilizando de monitorização ambulatorial contínua. Rev Dor. 2011;12(4):291-6.

8. Kaye AD, Baluch A, Scott JT. Pain management in the elderly population: a review. Oschser J. 2010;10(3):17987.

9. Barbosa REC, Assunção AA, Araújo TM. Distúrbios musculoesqueléticos em trabalhadores do setor saúde de Belo Horizonte, Minas Gerais, Brasil. Cad Saúde Pública. 2012;28(8):1569-1580

10. Santos CC, Pereira LSM, Resende MA, Magno F, Aguiar V. Aplicação da versão brasileira do questionário de dor McGuill em idosos com dor crônica. Acta Fisiátrica. 2006;13(2):75-82.

11. Kazanowiski MK, Laccetti MS. Dor fundamentos e abordagem clínica e tratamento. Rio de Janeiro: Guanabara Koogan; 2005.

12. Ministério da Previdência Social (BR). Proteção para o trabalhador e sua família. Brasília: Ministério da
Previdência Social; 2013 [acesso em 2013 Mar 01]. Disponível em: http://www.previdenciasocial.gov.br/

13. De Vitta A, Bertaglia RS, Padovani CR. Efeito de dois procedimentos educacionais sobre os sintomas musculoesqueléticos em trabalhadores administrativos. Rev Bras Fisioter. 2008;12(1):20-5.

14. Pimenta CAM, Teixeira MJ. Questionário de dor McMagill: Proposta de adaptação para a língua portuguesa. Rev Esc Enf. 1996;30(3):473-83.

15. Santos JPM, Hernandez CM. Atividade laboral sentada provoca queixas nos membros superiores e na coluna torácica. Rev Hórus. 2010;4(2):172-6.

16. Maciel ACC, Fernandes MB, Medeiros LS. Prevalência e fatores associados à sintomatologia dolorosa entre profissionais da indústria têxtil. Rev Bras Epidemiol. 2006;9(1):94-102.

17. Magee DJ. Avaliação musculoesquelética. $4^{\mathrm{a}}$ ed. São Paulo: Manole; 2005.

18. Cecilio HPM, Costa MAR, Silva RLD, Marcon SS. Condições de saúde da mulher trabalhadora na indústria do vestuário. Rev Rene. 2013;14(2):372-84.

19. Leone ET, Baltar P. A mulher na recuperação recente do mercado de trabalho brasileiro. Rev Bras Estud Popul. 2008;25(2):233-49.

20. Hoffmann R, Leone ET. Participação da mulher no mercado de trabalho e desigualdade de renda domiciliar per capta no Brasil: 1981-2002. Nova Economia. 2004;14(2):35-58.

21. Bruschini MCA. Trabalho e gênero no Brasil nos últimos dez anos. Cad Pesqui. 2007;37(132):537-72.

22. Morguchi CS, Alem MER, Coury HJCG. Evaluation of workload among industrial workers with the Need for Recovery Scale. Rev Bras Fisioter. 2011;15(2):154-9.

23. Pedrosa CM. Trabalho e saúde: uma reflexão sobre o processo produtivo na indústria de confecção. In: XXVII Congresso Internacional da Associação LatinoAmericana de Sociologia; 2011; Recife; 2011.

24. Lacerda JT, Ribeiro JD, Ribeiro DM, Traebert J. Prevalência da dor orofacial e seu impacto no desempenho diário em trabalhadores das indústrias têxteis do município de Laguna, SC. Ciênc Saúde Coletiva. 2011;16(10):4275-82.

25. Garcia AC Junior. Condições de trabalho e saúde dos trabalhadores na indústria do vestuário em Colatina - ES [dissertação]. Vitória: Universidade Federal do Espírito Santo; 2006. 
26. Cardoso AC, Batista MLL, Sandoval RA. Perfil epidemiológico e fatores relacionados à lombalgia em um grupo de costureiras da empresa têxtil Marka da Paz. EFDeportes.com. 2011;16(162):1-10.

27. Praia DT, Arêas GPT, Arêas FZS, Leite HR, Freire RC Júnior. Risco ergonômico em costureiras da indústria de confecções de Coari-AM. Rev Pesquisa em Fisioterapia. 2013;3(2):107-17.

28. Mascarenhas CHM, Santos LS. Avaliação da dor e da capacidade funcional em indivíduos com lombalgia crônica. J Health Sci Inst. 2011;29(3):205-8.

29. Oliveira MAS, Campos RS, Daher SS. Impacto do exercício na dor crônica. Rev Bras Med Esporte. 2014;20(3):200-203.

30. Yeng LT, Stump P, Kaziyama HHS, Fernandes MM. Medicina física e reabilitação em doentes com dor crônica. Rev Med. 2001;80(2):245-55.

31. Andersen LL, Christensen KB, Holtermann A, Poulsen OM, Sjogaard G, Pedersen MT, et al. Effect of physical exercise interventions on musculoskeletal pain in all body regions among Office workers: a one-year randomized controlled trial. Man Ther. 2010;15(1): 100-4.

32. Martignago CS, Depiné PJ. Acidentes e doenças do trabalho na indústria têxtil do Alto Vale do Itajaí. Base na indústria têxtil Rioestense. Caminhos. 2008;1(9):927.

33. Vitta A, Canonici AA, Conti MHS, Simeão SFAP. Prevalência e fatores associados a dor musculoesquelética em profissionais em profissionais de atividades sedentárias. Fisioter Mov. 2012;25(2): 273-80.
34. Freitas FCT, Barbosa LH, Alves LA, Marziale MHP, Robazzi MLCC. Avaliação Cinesiológica e Sintomatológica de Membros Inferiores de Costureiros Industriais. Rev Enferm UERJ. 2009;17(2):170-5.

35. Emmerich LBB. Análise das queixas musculoesqueléticas de trabalhadores de uma indústria têxtil da região metropolitana de porto alegre que receberam tratamento quiroprático [dissertação]. Novo Hamburgo: Universidade FEEVALE; 2011.

36. Oliveira CCES, Oliveira NMLO, Silva MD, Caldeira LC, Silva FS. Estudo da localização e caracterização da dor na coluna cervical e nos membros superiores de costureiras de uma microempresa. Coleç Pesqui Educ Fís. 2010;9(6):137-44.

37. Batista AGL, Vasconcelos LAP Principais queixas dolorosas em pacientes que procuram clínica de fisioterapia. Rev Dor. 2011;12(2):125-30.

\section{Endereço do primeiro autor:}

Marina Cauz

Universidade Comunitária da Região de Chapecó UNOCHAPECÓ

Av. Senador Atíllio Santana, 591 E

Bairro: Engenho Braun

CEP 89809-000 - Chapecó - SC - Brasil

E-mail:marinacauz@unochapeco.edu.br

\section{Endereço para correspondência:}

Josiane Schadeck de Almeida Altemar Universidade Comunitária da Região de Chapecó UNOCHAPECÓ

Av. Senador Atíllio Santana, $591 \mathrm{E}$

Bairro: Engenho Braun

CEP 89809-000 - Chapecó - SC - Brasil

E-mail: jsa_fisio@yahoo.com.br 